\title{
Stereoselectivity Control by Torsional Steering in an Intramolecular Diels-Alder Reaction of Vinyl Oxocarbenium Ions
}

Robert G. Iafe and K. N. Houk

\section{Supporting Information}

All ground state structures were fully optimized at the B3LYP/6-31+G* level of theory with Gaussian $03^{1}$. Transition structures $\mathbf{7}-\mathbf{1 4}$ were first optimized with HF level of theory. Oxocarbenium ring structures were truncated from optimized transition structures. All transition structures were validated as first-degree saddle points using frequency analysis. All zero-point energy corrections are reported unscaled.

\footnotetext{
${ }^{1}$ Gaussian 03, Revision C.02, M. J. Frisch, G. W. Trucks, H. B. Schlegel, G. E. Scuseria, M. A. Robb, J. R. Cheeseman, J. A. Montgomery, Jr., T. Vreven, K. N. Kudin, J. C. Burant, J. M. Millam, S. S. Iyengar, J. Tomasi, V. Barone, B. Mennucci, M. Cossi, G. Scalmani, N. Rega, G. A. Petersson, H. Nakatsuji, M. Hada, M. Ehara, K. Toyota, R. Fukuda, J. Hasegawa, M. Ishida, T. Nakajima, Y. Honda, O. Kitao, H. Nakai, M. Klene, X. Li, J. E. Knox, H. P. Hratchian, J. B. Cross, C. Adamo, J. Jaramillo, R. Gomperts, R. E. Stratmann, O. Yazyev, A. J. Austin, R. Cammi, C. Pomelli, J. W. Ochterski, P. Y. Ayala, K. Morokuma, G. A. Voth, P. Salvador, J. J. Dannenberg, V. G. Zakrzewski, S. Dapprich, A. D. Daniels, M. C. Strain, O.

Farkas, D. K. Malick, A. D. Rabuck, K. Raghavachari, J. B. Foresman, J. V. Ortiz, Q. Cui, A. G. Baboul, S. Clifford, J. Cioslowski, B. B. Stefanov, G. Liu, A. Liashenko, P. Piskorz, I. Komaromi, R. L. Martin, D. J. Fox, T. Keith, M. A. Al-Laham, C. Y. Peng, A. Nanayakkara, M. Challacombe, P. M. W. Gill, B. Johnson, W. Chen, M. W. Wong, C. Gonzalez, and J. A. Pople, Gaussian, Inc., Wallingford CT, 2004.
} 
Transition structure geometries and energies. Energies are in Hartrees, distances and coordinates in units of Angstroms.

\section{7}

\begin{tabular}{|c|c|c|c|}
\hline $\mathrm{C}$ & -2.389701 & -0.447895 & 1.192484 \\
\hline C & -1.085014 & 0.250399 & 0.897853 \\
\hline $\mathrm{C}$ & 0.112539 & -0.148825 & 1.484766 \\
\hline $\mathrm{C}$ & -0.675774 & -2.187049 & -0.421864 \\
\hline C & -2.156917 & -2.385208 & -0.507561 \\
\hline $\mathrm{C}$ & -2.969317 & -1.129466 & -0.067917 \\
\hline $\mathrm{H}$ & -0.190601 & -2.597286 & 0.458998 \\
\hline C & 1.463094 & 0.188544 & 1.169671 \\
\hline $\mathrm{C}$ & 2.178240 & -1.364053 & 0.029850 \\
\hline C & 1.467055 & -1.256809 & -1.180458 \\
\hline $\mathrm{H}$ & 1.976377 & -0.766087 & -2.009713 \\
\hline C & 0.114148 & -1.608928 & -1.387075 \\
\hline $\mathrm{H}$ & -0.340012 & -1.325316 & -2.335380 \\
\hline $\mathrm{H}$ & 2.151931 & -0.124351 & 1.954065 \\
\hline $\mathrm{O}$ & -1.265891 & 1.248777 & 0.042539 \\
\hline $\mathrm{C}$ & 1.907968 & 1.485104 & 0.527339 \\
\hline $\mathrm{H}$ & 2.925868 & 1.368310 & 0.141685 \\
\hline $\mathrm{H}$ & 1.998682 & 2.212077 & 1.349918 \\
\hline $\mathrm{C}$ & 1.007146 & 2.078451 & -0.554124 \\
\hline $\mathrm{H}$ & 1.473371 & 3.003088 & -0.915206 \\
\hline $\mathrm{H}$ & 0.927913 & 1.414351 & -1.423187 \\
\hline C & -0.396781 & 2.429487 & -0.068720 \\
\hline $\mathrm{H}$ & -0.010742 & -0.924079 & 2.236533 \\
\hline $\mathrm{H}$ & 1.815439 & -2.104895 & 0.739802 \\
\hline $\mathrm{C}$ & 3.686033 & -1.220166 & 0.022178 \\
\hline $\mathrm{H}$ & 4.028692 & -0.477125 & -0.705048 \\
\hline $\mathrm{H}$ & 4.081260 & -0.952255 & 1.007448 \\
\hline $\mathrm{H}$ & 4.138631 & -2.181113 & -0.253432 \\
\hline $\mathrm{H}$ & -2.441261 & -3.232755 & 0.125511 \\
\hline $\mathrm{H}$ & -2.444454 & -2.630218 & -1.538013 \\
\hline $\mathrm{H}$ & -2.997663 & -0.400364 & -0.884012 \\
\hline $\mathrm{H}$ & -2.254192 & -1.178238 & 1.996998 \\
\hline $\mathrm{H}$ & -3.106562 & 0.306128 & 1.540818 \\
\hline $\mathrm{H}$ & -4.003156 & -1.434583 & 0.124136 \\
\hline $\mathrm{H}$ & -0.349675 & 2.854313 & 0.942159 \\
\hline $\mathrm{C}$ & -1.142051 & 3.372329 & -1.001401 \\
\hline $\mathrm{H}$ & -2.161006 & 3.547028 & -0.644640 \\
\hline $\mathrm{H}$ & -0.623608 & 4.335628 & -1.043828 \\
\hline $\mathrm{H}$ & -1.192114 & 2.962040 & -2.016050 \\
\hline
\end{tabular}

Summary of Energies (298.15 K): $\mathrm{E}(0 \mathrm{~K}) \quad$ Energy Enthalpy Free Energy $\begin{array}{llll}-660.039444 & -660.390932 & -660.023102 & -660.080451\end{array}$ 


\section{8}

$\begin{array}{lrrr}\mathrm{C} & -2.455909 & 0.511565 & 0.960172 \\ \mathrm{C} & -1.006314 & 0.725907 & 0.598137 \\ \mathrm{C} & 0.023086 & 0.139784 & 1.331195 \\ \mathrm{C} & -1.313301 & -1.966900 & -0.114976 \\ \mathrm{C} & -2.791357 & -1.758682 & -0.234039 \\ \mathrm{C} & -3.209924 & -0.260676 & -0.146646 \\ \mathrm{H} & -0.954102 & -2.290024 & 0.857744 \\ \mathrm{C} & 1.414821 & 0.004607 & 1.045254 \\ \mathrm{C} & 1.659046 & -1.886515 & 0.298180 \\ \mathrm{C} & 0.995644 & -1.865380 & -0.944342 \\ \mathrm{H} & 1.614223 & -1.738532 & -1.832636 \\ \mathrm{C} & -0.403227 & -1.865524 & -1.140839 \\ \mathrm{H} & -0.769348 & -1.690140 & -2.151391 \\ \mathrm{H} & 1.979765 & -0.308653 & 1.923174 \\ \mathrm{O} & -0.901689 & 1.505097 & -0.469848 \\ \mathrm{C} & 2.212014 & 0.961005 & 0.185106 \\ \mathrm{H} & 3.163346 & 0.494174 & -0.090138 \\ \mathrm{H} & 2.492278 & 1.799055 & 0.840614 \\ \mathrm{C} & 1.529732 & 1.500274 & -1.070310 \\ \mathrm{H} & 2.237719 & 2.153581 & -1.596010 \\ \mathrm{H} & 1.296337 & 0.685723 & -1.766320 \\ \mathrm{C} & 0.264384 & 2.323078 & -0.839676 \\ \mathrm{H} & -0.064438 & 2.715061 & -1.805153 \\ \mathrm{C} & 0.394887 & 3.479273 & 0.148281 \\ \mathrm{H} & 1.210246 & 4.139685 & -0.167253 \\ \mathrm{H} & -0.527055 & 4.067681 & 0.168114 \\ \mathrm{H} & 0.608405 & 3.137010 & 1.165857 \\ \mathrm{H} & -0.323859 & -0.379813 & 2.220456 \\ \mathrm{H} & 1.109860 & -2.325849 & 1.128917 \\ \mathrm{C} & 3.147242 & -2.166820 & 0.341462 \\ \mathrm{H} & 3.677853 & -1.723380 & -0.507249 \\ \mathrm{H} & 3.608586 & -1.807250 & 1.267092 \\ \mathrm{H} & 3.312523 & -3.250847 & 0.301332 \\ \mathrm{H} & -3.296304 & -2.316846 & 0.561775 \\ \mathrm{H} & -3.149373 & -2.153962 & -1.193499 \\ \mathrm{H} & -3.032006 & 0.233278 & -1.107382 \\ \mathrm{H} & -2.533307 & -0.014635 & 1.917289 \\ \mathrm{H} & -2.925491 & 1.495784 & 1.079073 \\ \mathrm{H} & -287286 & -0.211839 & 0.042628\end{array}$

Summary of Energies (298.15 K):

$\mathrm{E}(0 \mathrm{~K})$

$-660.036728$

Energy

$-660.388540$

Enthalpy

$-660.020468$

Free Energy

$-660.077590$ 


$\begin{array}{rrrr}\mathrm{C} & 1.605868 & 1.834271 & 1.074153 \\ \mathrm{C} & 1.061523 & 0.453336 & 0.803303 \\ \mathrm{C} & -0.166814 & 0.048120 & 1.312066 \\ \mathrm{C} & -0.687556 & 1.934790 & -0.804400 \\ \mathrm{C} & 0.239916 & 3.097484 & -0.703281 \\ \mathrm{C} & 1.651649 & 2.697011 & -0.211292 \\ \mathrm{H} & -0.340282 & 1.090342 & -1.396978 \\ \mathrm{C} & -0.990003 & -1.091006 & 1.043981 \\ \mathrm{C} & -2.347566 & -0.574228 & -0.416392 \\ \mathrm{C} & -2.743682 & 0.751053 & -0.139112 \\ \mathrm{H} & -3.708185 & 0.879040 & 0.353217 \\ \mathrm{C} & -1.961364 & 1.911862 & -0.279633 \\ \mathrm{H} & -2.349666 & 2.828922 & 0.161541 \\ \mathrm{H} & -1.759627 & -1.236614 & 1.800573 \\ \mathrm{O} & 1.891870 & -0.231866 & 0.023069 \\ \mathrm{C} & -0.426654 & -2.406534 & 0.538929 \\ \mathrm{H} & -1.235522 & -3.047022 & 0.179409 \\ \mathrm{H} & -0.035967 & -2.924724 & 1.428471 \\ \mathrm{C} & 0.676538 & -2.339497 & -0.518634 \\ \mathrm{H} & 0.914514 & -3.366400 & -0.820822 \\ \mathrm{H} & 0.341206 & -1.828740 & -1.431267 \\ \mathrm{C} & 1.972871 & -1.697196 & -0.033247 \\ \mathrm{H} & -0.613757 & 0.794919 & 1.960130 \\ \mathrm{H} & -1.595762 & -0.694765 & -1.196055 \\ \mathrm{C} & -3.439441 & -1.628351 & -0.406186 \\ \mathrm{H} & -4.104462 & -1.509192 & 0.456195 \\ \mathrm{H} & -4.050595 & -1.528532 & -1.312110 \\ \mathrm{H} & -3.052827 & -2.649362 & -0.401201 \\ \mathrm{H} & -0.190093 & 3.866149 & -0.050698 \\ \mathrm{H} & 0.351923 & 3.548483 & -1.702539 \\ \mathrm{H} & 2.186274 & 2.146868 & -0.993343 \\ \mathrm{H} & 1.001676 & 2.331696 & 1.839567 \\ \mathrm{H} & 2.626356 & 1.730558 & 1.461981 \\ \mathrm{H} & 2.226333 & 3.608145 & -0.015628 \\ \mathrm{H} & 2.186932 & -2.017615 & 0.994548 \\ \mathrm{C} & 3.165874 & -1.986286 & -0.932011 \\ \mathrm{H} & 4.054633 & -1.455615 & -0.579104 \\ \mathrm{H} & 3.381958 & -3.059492 & -0.925362 \\ \mathrm{H} & 2.963012 & -1.681086 & -1.964535\end{array}$

Summary of Energies (298.15 $\mathrm{E}(0 \mathrm{~K})$

$-660.037583$

Energy

$-660.388430$
K) :

Enthalpy $-660.021091$
Free Energy $-660.079144$ 


\section{0}

$\begin{array}{rrrr}\mathrm{C} & 0.439087 & -2.466456 & 0.858968 \\ \mathrm{C} & -0.337746 & -1.217189 & 0.522587 \\ \mathrm{C} & -0.063407 & -0.010914 & 1.158635 \\ \mathrm{C} & 2.109735 & -0.516940 & -0.633811 \\ \mathrm{C} & 2.531884 & -1.944586 & -0.545718 \\ \mathrm{C} & 1.345493 & -2.911591 & -0.315523 \\ \mathrm{H} & 1.330235 & -0.286794 & -1.357738 \\ \mathrm{C} & -0.492216 & 1.331109 & 0.911263 \\ \mathrm{C} & 0.913363 & 2.244605 & -0.279235 \\ \mathrm{C} & 2.171347 & 1.805548 & 0.186018 \\ \mathrm{H} & 2.727113 & 2.497517 & 0.819568 \\ \mathrm{C} & 2.710247 & 0.512700 & 0.058363 \\ \mathrm{H} & 3.600711 & 0.285335 & 0.643046 \\ \mathrm{H} & -0.305243 & 1.991494 & 1.756768 \\ \mathrm{O} & -1.217584 & -1.464468 & -0.441431 \\ \mathrm{C} & -1.802228 & 1.646110 & 0.214156 \\ \mathrm{H} & -1.830089 & 2.700195 & -0.072144 \\ \mathrm{H} & -2.584657 & 1.549320 & 0.981000 \\ \mathrm{C} & -2.161361 & 0.788094 & -0.999762 \\ \mathrm{H} & -3.076708 & 1.193567 & -1.448883 \\ \mathrm{H} & -1.391918 & 0.863671 & -1.778895 \\ \mathrm{C} & -2.434714 & -0.688448 & -0.720701 \\ \mathrm{H} & -2.762678 & -1.153018 & -1.653803 \\ \mathrm{C} & -3.462688 & -0.979069 & 0.369056 \\ \mathrm{H} & -4.400066 & -0.460597 & 0.138471 \\ \mathrm{H} & -3.672456 & -2.051592 & 0.415539 \\ \mathrm{H} & -3.125549 & -0.652006 & 1.357840 \\ \mathrm{H} & 0.687608 & -0.116213 & 1.934518 \\ \mathrm{H} & 0.533011 & 1.742065 & -1.168319 \\ \mathrm{C} & 0.647585 & 3.737731 & -0.221461 \\ \mathrm{H} & 0.940187 & 4.160386 & 0.746007 \\ \mathrm{H} & 1.238181 & 4.243502 & -0.995714 \\ \mathrm{H} & -0.396813 & 3.996548 & -0.406766 \\ \mathrm{H} & 3.284240 & -2.065199 & 0.242392 \\ \mathrm{H} & 3.012737 & -2.229200 & -1.495653 \\ \mathrm{H} & 0.739308 & -2.994250 & -1.224366 \\ \mathrm{H} & 1.041810 & -2.303387 & 1.758028 \\ \mathrm{H} & -0.276958 & -3.269129 & 1.071573 \\ \mathrm{H} & 1.742383 & -3.910688 & -0.108024\end{array}$

Summary of Energies (298.15 K):

$\mathrm{E}(0 \mathrm{~K})$

$-660.034855 \quad-660.386255$

Enthalpy

$-660.018522$
Free Energy

$-660.075970$ 


$\begin{array}{rrrr}\mathrm{C} & 2.453054 & 0.539210 & -1.147060 \\ \mathrm{C} & 0.989773 & 0.738014 & -0.832268 \\ \mathrm{C} & 0.007782 & -0.043722 & -1.445468 \\ \mathrm{C} & 1.388292 & -1.824918 & 0.272071 \\ \mathrm{C} & 2.857434 & -1.536277 & 0.344477 \\ \mathrm{C} & 3.214631 & -0.048177 & 0.064777 \\ \mathrm{H} & 1.044236 & -2.327662 & -0.626807 \\ \mathrm{C} & -1.380105 & -0.188787 & -1.166285 \\ \mathrm{C} & -1.584398 & -1.942269 & -0.116801 \\ \mathrm{C} & -0.920871 & -1.692819 & 1.099000 \\ \mathrm{H} & -1.540114 & -1.427339 & 1.956060 \\ \mathrm{C} & 0.476010 & -1.603230 & 1.279169 \\ \mathrm{H} & 0.837394 & -1.247954 & 2.243139 \\ \mathrm{H} & -1.900982 & -0.678698 & -1.987251 \\ \mathrm{O} & 0.840812 & 1.695885 & 0.063473 \\ \mathrm{C} & -2.221140 & 0.892967 & -0.494587 \\ \mathrm{C} & -1.554211 & 2.274440 & -0.489126 \\ \mathrm{C} & -0.373017 & 2.439590 & 0.458844 \\ \mathrm{H} & 0.402658 & -0.689672 & -2.224421 \\ \mathrm{H} & -1.028026 & -2.504973 & -0.864164 \\ \mathrm{C} & -3.070838 & -2.235015 & -0.112183 \\ \mathrm{H} & -3.617302 & -1.585032 & 0.579281 \\ \mathrm{H} & -3.511569 & -2.129171 & -1.108410 \\ \mathrm{H} & -3.239616 & -3.271340 & 0.206417 \\ \mathrm{H} & 3.382436 & -2.170791 & -0.377677 \\ \mathrm{H} & 3.237537 & -1.791104 & 1.342649 \\ \mathrm{H} & 2.998083 & 0.562003 & 0.948042 \\ \mathrm{H} & 2.567358 & -0.108699 & -2.022152 \\ \mathrm{H} & 2.886274 & 1.516276 & -1.391728 \\ \mathrm{H} & 4.292725 & 0.022786 & -0.113795 \\ \mathrm{H} & -2.498562 & 0.618233 & 0.526836 \\ \mathrm{H} & -3.166949 & 0.967209 & -1.042331 \\ \mathrm{H} & -0.019256 & 3.472333 & 0.378722 \\ \mathrm{H} & -2.295206 & 3.020504 & -0.174923 \\ \mathrm{H} & -1.252572 & 2.556088 & -1.504959 \\ \mathrm{H} & -0.643472 & 2.134326 & 1.926387 \\ \mathrm{H} & -1.437182 & 2.793397 & 2.295627 \\ \mathrm{H} & 0.954865 & 1.097310 & 2.083268 \\ \mathrm{H} & -0.253471 & 2.319224 & 2.525118\end{array}$

Summary of Energies ( $298.15 \mathrm{~K})$ :

$\begin{array}{llll}\mathrm{E}(\mathrm{O} \mathrm{K}) & \text { Energy } & \text { Enthalpy } & \text { Free Energy } \\ -660.031224 & -660.382637 & -660.014753 & -660.072704\end{array}$ 


$\begin{array}{rrrr}\mathrm{C} & 2.405110 & -0.099187 & -1.229977 \\ \mathrm{C} & 1.025150 & 0.378782 & -0.850324 \\ \mathrm{C} & -0.116810 & -0.180069 & -1.426617 \\ \mathrm{C} & 0.933169 & -2.092518 & 0.355350 \\ \mathrm{C} & 2.432280 & -2.129443 & 0.371025 \\ \mathrm{C} & 3.100161 & -0.780503 & -0.029076 \\ \mathrm{H} & 0.456488 & -2.569426 & -0.495606 \\ \mathrm{C} & -1.499622 & -0.021421 & -1.114507 \\ \mathrm{C} & -2.006575 & -1.611744 & 0.040626 \\ \mathrm{C} & -1.265850 & -1.436597 & 1.228014 \\ \mathrm{H} & -1.787652 & -0.993479 & 2.076597 \\ \mathrm{C} & 0.122609 & -1.627652 & 1.371347 \\ \mathrm{H} & 0.584668 & -1.304459 & 2.303273 \\ \mathrm{H} & -2.127555 & -0.443003 & -1.897958 \\ \mathrm{O} & 1.112490 & 1.347150 & 0.044725 \\ \mathrm{C} & -2.094632 & 1.260213 & -0.531748 \\ \mathrm{C} & -1.107443 & 2.416162 & -0.338644 \\ \mathrm{C} & 0.015077 & 2.124581 & 0.649884 \\ \mathrm{H} & 0.111372 & -0.914503 & -2.193768 \\ \mathrm{H} & -1.602255 & -2.325178 & -0.675180 \\ \mathrm{C} & -3.521156 & -1.611349 & 0.101105 \\ \mathrm{H} & -3.909038 & -0.840461 & 0.775079 \\ \mathrm{H} & -3.969218 & -1.464914 & -0.886698 \\ \mathrm{H} & -3.871687 & -2.581426 & 0.474942 \\ \mathrm{H} & 2.773273 & -2.913004 & -0.314062 \\ \mathrm{H} & 2.785448 & -2.395349 & 1.376132 \\ \mathrm{H} & 3.088735 & -0.091647 & 0.821763 \\ \mathrm{H} & 2.348712 & -0.781001 & -2.084320 \\ \mathrm{H} & 3.001441 & 0.770257 & -1.532289 \\ \mathrm{H} & 4.151037 & -0.969640 & -0.271518 \\ \mathrm{H} & -2.603396 & 1.065691 & 0.418488 \\ \mathrm{H} & -2.883243 & 1.591081 & -1.218365 \\ \mathrm{H} & -1.666640 & 3.277391 & 0.045675 \\ \mathrm{H} & -0.676447 & 2.733861 & -1.296387 \\ \mathrm{H} & -0.352191 & 1.513053 & 1.481671 \\ \mathrm{C} & 0.691662 & 3.375125 & 1.192748 \\ \mathrm{H} & 1.046961 & 4.013712 & 0.376842 \\ \mathrm{H} & -0.021079 & 3.946838 & 1.796061 \\ \mathrm{H} & 1.543575 & 3.112115 & 1.826587\end{array}$

Summary of Energies ( 298.15 K):

$\begin{array}{llll}\mathrm{E}(0 \mathrm{~K}) & \text { Energy } & \text { Enthalpy } & \text { Free Energy } \\ -660.034418 & -660.385677 & -660.017930 & -660.075816\end{array}$ 


$\begin{array}{rrrr}\mathrm{C} & -2.383904 & -0.863650 & -1.031715 \\ \mathrm{C} & -0.903192 & -0.923582 & -0.743590 \\ \mathrm{C} & -0.040631 & 0.037946 & -1.269114 \\ \mathrm{C} & -1.503546 & 1.412803 & 0.705320 \\ \mathrm{C} & -2.950399 & 1.067324 & 0.578941 \\ \mathrm{C} & -3.192323 & -0.414289 & 0.212504 \\ \mathrm{H} & -0.909081 & 0.768213 & 1.349987 \\ \mathrm{C} & 1.340419 & 0.324206 & -1.049619 \\ \mathrm{C} & 1.467465 & 1.805291 & 0.370235 \\ \mathrm{C} & 0.464569 & 2.743001 & 0.049634 \\ \mathrm{H} & 0.781452 & 3.648145 & -0.468832 \\ \mathrm{C} & -0.922987 & 2.540721 & 0.164128 \\ \mathrm{H} & -1.573415 & 3.268638 & -0.319472 \\ \mathrm{H} & 1.751851 & 0.958557 & -1.831382 \\ \mathrm{O} & -0.632753 & -1.944794 & 0.050886 \\ \mathrm{C} & 2.326887 & -0.734816 & -0.557286 \\ \mathrm{C} & 1.786642 & -2.169504 & -0.587956 \\ \mathrm{C} & 0.685163 & -2.495065 & 0.414522 \\ \mathrm{H} & -0.553476 & 0.726543 & -1.931169 \\ \mathrm{H} & 1.221272 & 1.099654 & 1.162890 \\ \mathrm{C} & 2.909451 & 2.273868 & 0.354341 \\ \mathrm{H} & 3.114497 & 2.908169 & -0.515030 \\ \mathrm{H} & 3.114918 & 2.867600 & 1.254012 \\ \mathrm{H} & 3.622641 & 1.445879 & 0.349749 \\ \mathrm{H} & -3.431841 & 1.727882 & -0.151762 \\ \mathrm{H} & -3.441057 & 1.253855 & 1.548384 \\ \mathrm{H} & -2.936153 & -1.062590 & 1.058364 \\ \mathrm{H} & -2.575144 & -0.184650 & -1.868622 \\ \mathrm{H} & -2.718753 & -1.865293 & -1.324413 \\ \mathrm{H} & -4.259963 & -0.559345 & 0.017321 \\ \mathrm{H} & 2.692155 & -0.516826 & 0.451306 \\ \mathrm{H} & 3.212492 & -0.686094 & -1.200689 \\ \mathrm{H} & 2.615101 & -2.851639 & -0.358238 \\ \mathrm{H} & 1.447870 & -2.432400 & -1.597197 \\ \mathrm{H} & 0.483616 & -3.568752 & 0.353787 \\ \mathrm{C} & 0.964044 & -2.138541 & 1.869972 \\ \mathrm{H} & 1.102791 & -1.062880 & 2.020778 \\ \mathrm{H} & 0.139263 & -2.470794 & 2.507283 \\ \mathrm{H} & 1.875041 & -2.647796 & 2.203959\end{array}$

Summary of Energies ( 298.15 K):

$\begin{array}{llll}E(0 \mathrm{~K}) & \text { Energy } & \text { Enthalpy } & \text { Free Energy } \\ -660.031856 & -660.383167 & -660.015467 & -660.073082\end{array}$




$\begin{array}{rrrr}\mathrm{C} & 1.999303 & 1.404079 & 1.100203 \\ \mathrm{C} & 1.142156 & 0.214042 & 0.749609 \\ \mathrm{C} & -0.150273 & 0.092329 & 1.253947 \\ \mathrm{C} & -0.259626 & 2.063912 & -0.745026 \\ \mathrm{C} & 0.869672 & 3.023000 & -0.555910 \\ \mathrm{C} & 2.186235 & 2.339514 & -0.121860 \\ \mathrm{H} & -0.065101 & 1.204319 & -1.383866 \\ \mathrm{C} & -1.204247 & -0.843091 & 1.007823 \\ \mathrm{C} & -2.378180 & -0.088143 & -0.487809 \\ \mathrm{C} & -2.536360 & 1.287318 & -0.203304 \\ \mathrm{H} & -3.475849 & 1.585224 & 0.262718 \\ \mathrm{C} & -1.541919 & 2.276201 & -0.278781 \\ \mathrm{H} & -1.763085 & 3.238092 & 0.182342 \\ \mathrm{H} & -1.991370 & -0.764005 & 1.754473 \\ \mathrm{O} & 1.792297 & -0.592356 & -0.075197 \\ \mathrm{C} & -0.933515 & -2.292079 & 0.594976 \\ \mathrm{C} & 0.541003 & -2.672700 & 0.433224 \\ \mathrm{C} & 1.282057 & -1.857514 & -0.623131 \\ \mathrm{H} & -0.407929 & 0.912284 & 1.915354 \\ \mathrm{H} & -1.634178 & -0.338067 & -1.243552 \\ \mathrm{C} & -3.635855 & -0.935720 & -0.521365 \\ \mathrm{H} & -4.311939 & -0.678642 & 0.301274 \\ \mathrm{H} & -4.174047 & -0.758751 & -1.461101 \\ \mathrm{H} & -3.429620 & -2.007308 & -0.470556 \\ \mathrm{H} & 0.583523 & 3.799952 & 0.162744 \\ \mathrm{H} & 1.059802 & 3.531817 & -1.515241 \\ \mathrm{H} & 2.603649 & 1.758964 & -0.952309 \\ \mathrm{H} & 1.554973 & 1.953609 & 1.935638 \\ \mathrm{H} & 2.983843 & 1.042140 & 1.418590 \\ \mathrm{H} & 2.921944 & 3.112060 & 0.124640 \\ \mathrm{H} & 0.585497 & -3.726715 & 0.135606 \\ \mathrm{H} & 1.078908 & -2.599453 & 1.386642 \\ \mathrm{H} & 0.603784 & -1.576631 & -1.439928 \\ \mathrm{H} & -1.458205 & -2.548560 & -0.331702 \\ \mathrm{H} & -1.380094 & -2.934185 & 1.364099 \\ \mathrm{C} & 2.508886 & -2.555057 & -1.191459 \\ \mathrm{H} & 3.187464 & -2.864445 & -0.389286 \\ \mathrm{H} & 2.201466 & -3.445682 & -1.749096 \\ \mathrm{H} & 3.052168 & -1.893711 & -1.872733\end{array}$

Summary of Energies $(298.15 \quad \mathrm{~K})$ :

$\begin{array}{llll}E(0 \mathrm{~K}) & \text { Energy } & \text { Enthalpy } & \text { Free Energy } \\ -660.034855 & -660.386093 & -660.018475 & -660.076115\end{array}$


Unsubstituted transition structure geometries and energies. Energies are in Hartrees, distances and coordinates in units of Angstroms.

\section{Unsubstituted Endo Staggered TS}

$\begin{array}{rrrr}\mathrm{C} & -2.477893 & 0.560682 & 0.990376 \\ \mathrm{C} & -1.046932 & 0.886611 & 0.643948 \\ \mathrm{C} & 0.025182 & 0.365533 & 1.362589 \\ \mathrm{C} & -1.167504 & -1.789778 & -0.164790 \\ \mathrm{C} & -2.655650 & -1.683767 & -0.283809 \\ \mathrm{C} & -3.178008 & -0.222063 & -0.144225 \\ \mathrm{H} & -0.788466 & -2.115446 & 0.799530 \\ \mathrm{C} & 1.422083 & 0.338641 & 1.071548 \\ \mathrm{C} & 1.794415 & -1.525482 & 0.262929 \\ \mathrm{C} & 1.131622 & -1.497981 & -0.978095 \\ \mathrm{H} & 1.741051 & -1.295147 & -1.858600 \\ \mathrm{C} & -0.264546 & -1.587322 & -1.181079 \\ \mathrm{H} & -0.639267 & -1.400999 & -2.186431 \\ \mathrm{H} & 2.009394 & 0.033266 & 1.937144 \\ \mathrm{O} & -0.989635 & 1.708447 & -0.398960 \\ \mathrm{C} & 2.159330 & 1.363272 & 0.236447 \\ \mathrm{H} & 3.138840 & 0.965413 & -0.047820 \\ \mathrm{H} & 2.379768 & 2.200847 & 0.917184 \\ \mathrm{C} & 1.438820 & 1.907482 & -0.995567 \\ \mathrm{H} & 2.086722 & 2.650409 & -1.477886 \\ \mathrm{H} & 1.264265 & 1.121812 & -1.740054 \\ \mathrm{C} & 0.123697 & 2.600363 & -0.677643 \\ \mathrm{H} & -0.278821 & -0.202665 & 2.237804 \\ \mathrm{H} & 1.273291 & -2.021592 & 1.079458 \\ \mathrm{C} & 3.297329 & -1.704171 & 0.303570 \\ \mathrm{H} & 3.797842 & -1.203993 & -0.531637 \\ \mathrm{H} & 3.732417 & -1.339973 & 1.239941 \\ \mathrm{H} & 3.535455 & -2.773301 & 0.235492 \\ \mathrm{H} & -3.121661 & -2.304091 & 0.489349 \\ \mathrm{H} & -2.983061 & -2.067823 & -1.258500 \\ \mathrm{H} & -3.037079 & 0.316208 & -1.087160 \\ \mathrm{H} & -2.519853 & -0.001686 & 1.929030 \\ \mathrm{H} & -3.013471 & 1.506243 & 1.141837 \\ \mathrm{H} & -4.255665 & -0.254835 & 0.046339 \\ \mathrm{H} & 0.221733 & 3.274093 & 0.181707 \\ \mathrm{H} & -0.238027 & 3.173561 & -1.532246 \\ & & & \end{array}$

Summary of Energies ( 298.15 K):

$\begin{array}{llll}\mathrm{E}(0 \mathrm{~K}) & \text { Energy } & \text { Enthalpy } & \text { Free Energy } \\ -620.746568 & -621.070419 & -620.731718 & -620.785803\end{array}$




\section{Unsubstituted Endo Eclipsed TS}

$\begin{array}{rrrr}\mathrm{C} & 2.427457 & 0.706814 & -0.993252 \\ \mathrm{C} & 0.992762 & 0.909058 & -0.574279 \\ \mathrm{C} & -0.062030 & 0.346769 & -1.294143 \\ \mathrm{C} & 1.227019 & -1.747907 & 0.091051 \\ \mathrm{C} & 2.716745 & -1.592854 & 0.152480 \\ \mathrm{C} & 3.199245 & -0.114640 & 0.065151 \\ \mathrm{H} & 0.825596 & -2.089792 & -0.858086 \\ \mathrm{C} & -1.456991 & 0.252041 & -1.006139 \\ \mathrm{C} & -1.748054 & -1.601250 & -0.224565 \\ \mathrm{C} & -1.047413 & -1.582542 & 0.999306 \\ \mathrm{H} & -1.631777 & -1.401064 & 1.901730 \\ \mathrm{C} & 0.352808 & -1.616226 & 1.151045 \\ \mathrm{H} & 0.759116 & -1.440753 & 2.146315 \\ \mathrm{H} & -2.013852 & -0.075030 & -1.882887 \\ \mathrm{O} & 0.942271 & 1.667135 & 0.509721 \\ \mathrm{C} & -2.231146 & 1.288387 & -0.190711 \\ \mathrm{C} & -1.413173 & 2.497269 & 0.274411 \\ \mathrm{C} & -0.254372 & 2.145926 & 1.189556 \\ \mathrm{H} & 0.267843 & -0.172592 & -2.189483 \\ \mathrm{H} & -1.243919 & -2.087466 & -1.057756 \\ \mathrm{C} & -3.248711 & -1.814177 & -0.220284 \\ \mathrm{H} & -3.743868 & -1.259378 & 0.583475 \\ \mathrm{H} & -3.704729 & -1.525488 & -1.172492 \\ \mathrm{H} & -3.465736 & -2.878670 & -0.067118 \\ \mathrm{H} & 3.165428 & -2.163344 & -0.667710 \\ \mathrm{H} & 3.094911 & -2.015519 & 1.092671 \\ \mathrm{H} & 3.088054 & 0.372745 & 1.039151 \\ \mathrm{H} & 2.471099 & 0.219602 & -1.972368 \\ \mathrm{H} & 2.902259 & 1.691167 & -1.086460 \\ \mathrm{H} & 4.267761 & -0.109342 & -0.173573 \\ \mathrm{H} & -2.712993 & 0.829331 & 0.679094 \\ \mathrm{H} & -3.054215 & 1.646999 & -0.820526 \\ \mathrm{H} & 0.114139 & 3.026445 & 1.718685 \\ \mathrm{H} & -2.074902 & 3.166111 & 0.838746 \\ \mathrm{H} & -1.045217 & 3.079038 & -0.579482 \\ \mathrm{H} & -0.528037 & 1.389913 & 1.932552\end{array}$

Summary of Energies ( 298.15 K):

$\begin{array}{llll}E(0 \mathrm{~K}) & \text { Energy } & \text { Enthalpy } & \text { Free Energy } \\ -620.741279 & -621.064951 & -620.726254 & -620.781079\end{array}$


Model structure geometries and energies. Energies are in Hartrees, distances and coordinates in units of Angstroms.

\section{Table 1, Entry 1, Equatorial Methyl}

$\begin{array}{rrrr}\mathrm{C} & -2.578971 & -1.187459 & 0.071621 \\ \mathrm{C} & -1.349096 & -0.313938 & 0.040027 \\ \mathrm{C} & -1.430356 & 1.066102 & -0.123878 \\ \mathrm{C} & -0.450571 & 2.086602 & 0.065077 \\ \mathrm{H} & -0.773136 & 3.039767 & -0.353374 \\ \mathrm{O} & -0.248549 & -1.041915 & 0.181036 \\ \mathrm{C} & 1.043780 & 1.894883 & -0.082327 \\ \mathrm{H} & 1.565384 & 2.734551 & 0.388181 \\ \mathrm{H} & 1.255003 & 1.997753 & -1.158504 \\ \mathrm{C} & 1.634382 & 0.574749 & 0.409139 \\ \mathrm{H} & 2.717018 & 0.601495 & 0.236275 \\ \mathrm{H} & 1.498623 & 0.448887 & 1.490021 \\ \mathrm{C} & 1.083871 & -0.656041 & -0.306368 \\ \mathrm{H} & -2.437665 & 1.419513 & -0.328330 \\ \mathrm{H} & -3.467330 & -0.598571 & -0.179445 \\ \mathrm{H} & -2.463106 & -1.963743 & -0.695018 \\ \mathrm{H} & 0.953921 & -0.442729 & -1.375098 \\ \mathrm{C} & 1.939975 & -1.899430 & -0.118378 \\ \mathrm{H} & 1.476380 & -2.766609 & -0.597073 \\ \mathrm{H} & 2.923748 & -1.742234 & -0.572260 \\ \mathrm{H} & 2.081704 & -2.121037 & 0.945181 \\ \mathrm{H} & -2.700241 & -1.664529 & 1.021673\end{array}$

Summary of Energies (298.15 K): E $(0 \mathrm{~K})$ Energy Enthalpy Free Energy $\begin{array}{llll}-387.430634 & -387.625878 & -387.421044 & -387.463959\end{array}$

\section{Table 1, Entry 1, Axial Methyl}

$\begin{array}{lrrr}\mathrm{C} & 2.823710 & -0.160436 & -0.074858 \\ \mathrm{C} & 1.342915 & 0.130509 & -0.105481 \\ \mathrm{C} & 0.830956 & 1.354431 & 0.319362 \\ \mathrm{C} & -0.465349 & 1.930614 & 0.162626 \\ \mathrm{H} & -0.600095 & 2.803575 & 0.801233 \\ \mathrm{O} & 0.672363 & -0.908505 & -0.584528 \\ \mathrm{C} & -1.747481 & 1.137864 & 0.029376 \\ \mathrm{H} & -2.542044 & 1.793235 & -0.341948 \\ \mathrm{H} & -2.054604 & 0.880690 & 1.054121 \\ \mathrm{C} & -1.695900 & -0.129126 & -0.821778 \\ \mathrm{H} & -2.699010 & -0.573829 & -0.842387 \\ \mathrm{H} & -1.450821 & 0.111751 & -1.863081 \\ \mathrm{C} & -0.744274 & -1.219848 & -0.335939\end{array}$




$\begin{array}{rrrr}\mathrm{H} & -0.873310 & -2.092593 & -0.980598 \\ \mathrm{C} & -0.911963 & -1.647467 & 1.119705 \\ \mathrm{H} & -1.947866 & -1.960077 & 1.292035 \\ \mathrm{H} & -0.261531 & -2.498185 & 1.342891 \\ \mathrm{H} & -0.678625 & -0.840531 & 1.821562 \\ \mathrm{H} & 1.582253 & 2.006366 & 0.757163 \\ \mathrm{H} & 3.356416 & 0.641470 & 0.446794 \\ \mathrm{H} & 2.976057 & -1.088037 & 0.490543 \\ \mathrm{H} & 3.218597 & -0.295039 & -1.060174\end{array}$

Summary of Energies (298.15 K): $\mathrm{E}(0 \mathrm{~K}) \quad$ Energy Enthalpy Free Energy $\begin{array}{llll}-387.428702 & -387.624289 & -387.419238 & -387.461647\end{array}$

\section{Table 1, Entry 2, Equatorial Phenyl}

$\begin{array}{lrrr}\mathrm{C} & -2.311037 & 2.618936 & 0.204946 \\ \mathrm{C} & -2.128188 & 1.127525 & 0.078038 \\ \mathrm{C} & -3.172873 & 0.254524 & 0.198286 \\ \mathrm{C} & -3.236195 & -1.129022 & -0.157777 \\ \mathrm{H} & -4.133532 & -1.596481 & 0.208544 \\ \mathrm{O} & -0.875784 & 0.825287 & -0.177027 \\ \mathrm{C} & -2.073104 & -2.094443 & -0.116958 \\ \mathrm{H} & -2.328186 & -2.986256 & -0.674965 \\ \mathrm{H} & -2.015818 & -2.413553 & 0.923326 \\ \mathrm{C} & -0.700728 & -1.589959 & -0.544802 \\ \mathrm{H} & 0.009161 & -2.394376 & -0.389718 \\ \mathrm{H} & -0.668424 & -1.351813 & -1.602360 \\ \mathrm{C} & -0.222503 & -0.383563 & 0.254769 \\ \mathrm{H} & -4.107499 & 0.703129 & 0.480157 \\ \mathrm{H} & -3.325499 & 2.847957 & 0.509843 \\ \mathrm{H} & -1.641561 & 2.966233 & 0.985090 \\ \mathrm{H} & -0.473967 & -0.517073 & 1.300191 \\ \mathrm{C} & 1.263751 & -0.138287 & 0.132711 \\ \mathrm{C} & 2.089433 & -0.376139 & 1.221681 \\ \mathrm{C} & 1.827886 & 0.288538 & -1.066751 \\ \mathrm{C} & 3.463966 & -0.199586 & 1.117077 \\ \mathrm{H} & 1.668992 & -0.697530 & 2.159193 \\ \mathrm{C} & 3.195982 & 0.470804 & -1.171002 \\ \mathrm{H} & 1.201275 & 0.490161 & -1.917579 \\ \mathrm{C} & 4.018520 & 0.224215 & -0.078169 \\ \mathrm{H} & 4.092408 & -0.387120 & 1.968643 \\ \mathrm{H} & 3.622006 & 0.804315 & -2.100022 \\ \mathrm{H} & 5.080782 & 0.365586 & -0.160773 \\ \mathrm{H} & -2.063319 & 3.123272 & -0.705648 \\ & & & \end{array}$

Summary of Energies (298.15 K): 


$\begin{array}{llll}\text { E }(0 \mathrm{~K}) & \text { Energy } & \text { Enthalpy } & \text { Free Energy } \\ -579.111669 & -579.361196 & -579.100741 & -579.147801\end{array}$

\section{Table 1, Entry 2, Axial Phenyl}

$\begin{array}{lrrr}\mathrm{C} & 2.724435 & -2.137582 & -0.494877 \\ \mathrm{C} & 2.086699 & -0.809404 & -0.162122 \\ \mathrm{C} & 2.385998 & -0.152732 & 0.998664 \\ \mathrm{C} & 2.120553 & 1.195413 & 1.388907 \\ \mathrm{H} & 2.233672 & 1.342288 & 2.448982 \\ \mathrm{O} & 1.284446 & -0.446833 & -1.133886 \\ \mathrm{C} & 0.990396 & 2.026273 & 0.832896 \\ \mathrm{H} & 1.147965 & 3.065287 & 1.093813 \\ \mathrm{H} & 0.103142 & 1.717216 & 1.381099 \\ \mathrm{C} & 0.725018 & 1.909350 & -0.660665 \\ \mathrm{H} & -0.043637 & 2.626998 & -0.922602 \\ \mathrm{H} & 1.601255 & 2.183895 & -1.237473 \\ \mathrm{C} & 0.246977 & 0.543209 & -1.145911 \\ \mathrm{H} & 0.055216 & 0.630020 & -2.205347 \\ \mathrm{H} & 2.998106 & -0.716944 & 1.677458 \\ \mathrm{H} & 3.260981 & -2.523155 & 0.363877 \\ \mathrm{H} & 1.932133 & -2.837923 & -0.735675 \\ \mathrm{C} & -1.032577 & 0.046672 & -0.484769 \\ \mathrm{C} & -1.108449 & -1.097431 & 0.295266 \\ \mathrm{C} & -2.198755 & 0.778180 & -0.710562 \\ \mathrm{C} & -2.317523 & -1.491938 & 0.857804 \\ \mathrm{H} & -0.242002 & -1.704288 & 0.471676 \\ \mathrm{C} & -3.402585 & 0.387110 & -0.151518 \\ \mathrm{H} & -2.177232 & 1.651688 & -1.339529 \\ \mathrm{C} & -3.465497 & -0.751418 & 0.641474 \\ \mathrm{H} & -2.355437 & -2.382866 & 1.458269 \\ \mathrm{H} & -4.290181 & 0.962624 & -0.342201 \\ \mathrm{H} & -4.399895 & -1.059577 & 1.073697 \\ \mathrm{H} & 3.372206 & -2.054814 & -1.342487\end{array}$

Summary of Energies (298.15 K): $\begin{array}{llll}\mathrm{E}(0 \mathrm{~K}) & \text { Energy } & \text { Enthalpy } & \text { Free Energy } \\ -579.110348 & -579.360351 & -579.098737 & -579.147431\end{array}$

Table 1, Entry 3, Equatorial t-Butyl

$\begin{array}{lrrr}\mathrm{C} & 2.007358 & 2.456341 & 0.075578 \\ \mathrm{C} & 1.668941 & 0.986809 & 0.043673 \\ \mathrm{C} & 2.608522 & 0.033847 & -0.234645 \\ \mathrm{C} & 2.542758 & -1.381464 & -0.034911 \\ \mathrm{H} & 3.356863 & -1.892885 & -0.518448 \\ \mathrm{O} & 0.405755 & 0.781475 & 0.332722 \\ \mathrm{C} & 1.278892 & -2.211103 & -0.087806\end{array}$




$\begin{array}{rrrr}\mathrm{H} & 1.466209 & -3.174880 & 0.368177 \\ \mathrm{H} & 1.125259 & -2.418977 & -1.146066 \\ \mathrm{C} & -0.000215 & -1.606209 & 0.479716 \\ \mathrm{H} & -0.786710 & -2.335169 & 0.337462 \\ \mathrm{H} & 0.079362 & -1.441894 & 1.547684 \\ \mathrm{C} & -0.387633 & -0.298871 & -0.201348 \\ \mathrm{H} & 3.569804 & 0.415940 & -0.524748 \\ \mathrm{H} & 3.023343 & 2.615084 & -0.266561 \\ \mathrm{H} & 1.341967 & 2.963054 & -0.615728 \\ \mathrm{H} & -0.148728 & -0.356763 & -1.258252 \\ \mathrm{C} & -1.867487 & 0.130700 & -0.071417 \\ \mathrm{C} & -2.055605 & 1.499205 & -0.748797 \\ \mathrm{H} & -3.107226 & 1.763913 & -0.761400 \\ \mathrm{H} & -1.524952 & 2.285991 & -0.227910 \\ \mathrm{H} & -1.712023 & 1.481927 & -1.780051 \\ \mathrm{C} & -2.749352 & -0.891150 & -0.812457 \\ \mathrm{H} & -2.755107 & -1.865869 & -0.338328 \\ \mathrm{H} & -3.775481 & -0.541957 & -0.828942 \\ \mathrm{H} & -2.434432 & -1.018574 & -1.844739 \\ \mathrm{C} & -2.304190 & 0.222796 & 1.398234 \\ \mathrm{H} & -3.329210 & 0.572966 & 1.457715 \\ \mathrm{H} & -2.265534 & -0.740533 & 1.897002 \\ \mathrm{H} & -1.687246 & 0.920576 & 1.954607 \\ \mathrm{H} & 1.865866 & 2.870845 & 1.051829 \\ & & & \end{array}$

Summary of Energies (298.15 K): $\mathrm{E}(0 \mathrm{~K}) \quad$ Energy Enthalpy Free Energy $\begin{array}{llll}-505.282022 & -505.562648 & -505.270190 & -505.319015\end{array}$

\section{Table 1, Entry 3, Axial t-Butyl}

$\begin{array}{lrrr}\mathrm{C} & 2.277356 & -2.167261 & -0.099117 \\ \mathrm{C} & 1.646235 & -0.794803 & -0.126112 \\ \mathrm{C} & 2.232963 & 0.258207 & 0.523624 \\ \mathrm{C} & 1.971876 & 1.658361 & 0.431378 \\ \mathrm{H} & 2.423406 & 2.208225 & 1.239157 \\ \mathrm{O} & 0.559636 & -0.815779 & -0.853037 \\ \mathrm{C} & 0.633201 & 2.243932 & 0.049733 \\ \mathrm{H} & 0.763458 & 3.286753 & -0.212126 \\ \mathrm{H} & 0.043351 & 2.252460 & 0.960471 \\ \mathrm{C} & -0.126430 & 1.547962 & -1.069815 \\ \mathrm{H} & -1.018895 & 2.123625 & -1.281991 \\ \mathrm{H} & 0.462657 & 1.582057 & -1.980589 \\ \mathrm{C} & -0.561962 & 0.095748 & -0.889188 \\ \mathrm{H} & -1.008762 & -0.174527 & -1.835011 \\ \mathrm{C} & -1.615417 & -0.295330 & 0.188379 \\ \mathrm{H} & 3.081700 & -0.013456 & 1.123221\end{array}$




$\begin{array}{lrrr}\mathrm{H} & 3.096547 & -2.191755 & 0.609573 \\ \mathrm{H} & 1.526363 & -2.873756 & 0.237339 \\ \mathrm{C} & -2.168072 & -1.683189 & -0.189884 \\ \mathrm{H} & -1.389395 & -2.437227 & -0.203297 \\ \mathrm{H} & -2.918295 & -1.995896 & 0.528132 \\ \mathrm{H} & -2.637799 & -1.669524 & -1.168922 \\ \mathrm{C} & -2.774301 & 0.715475 & 0.134450 \\ \mathrm{H} & -3.159173 & 0.835320 & -0.874495 \\ \mathrm{H} & -3.593555 & 0.364665 & 0.751643 \\ \mathrm{H} & -2.488466 & 1.694174 & 0.505714 \\ \mathrm{C} & -1.079387 & -0.375845 & 1.626934 \\ \mathrm{H} & -0.311024 & -1.132540 & 1.735399 \\ \mathrm{H} & -0.682735 & 0.563078 & 1.991550 \\ \mathrm{H} & -1.891593 & -0.654876 & 2.289680 \\ \mathrm{H} & 2.608753 & -2.460113 & -1.073444\end{array}$

Summary of Energies (298.15 K):

$\mathrm{E}(0 \mathrm{~K})$

Energy

Enthalpy

Free Energy

$-505.276958$

$-505.558048$

$-505.265320$

$-505.312888$ 